\title{
A versatile low-cost interface module that features optical isolation
}

\author{
LOUIS A. CHIODO \\ Wayne State University, Detroit, Michigan 48202
}

The complete design and construction of a versatile low-cost interface module are presented. This module functions as a combination pulse lengthener-stretcher with an added feature of optical isolation between the input and output circuits. It is useful in interfacing many behavioral and physiological instruments that utilize different voltage levels and polarities in their respective logic systems. The cost of the components needed for module construction (without power supply) is approximately $\$ 15$.

It is sometimes necessary to interface two or more pieces of equipment in tandem that were not originally intended to operate in this manner. The problem frequently takes the form of a logistically strategic output pulse being too short in duration and/or inappropriate in amplitude to be reliably read by the next logic unit. This improper output pulse must be reliably modified by changing its length and increasing or decreasing its amplitude. This changes the signal into a properly referenced logic pulse that is appropriate for further system use.

Various means by which the interfacing of divergent pieces of equipment may be accomplished have been described in the literature. Level shifting circuits can take a voltage referred to some dc level and produce an amplified output that is referenced to ground, yet not "load" the input signal source (RCA, 1970; Widler, 1968). Pulse stretchers are frequently employed and have been constructed in various ways. Bliss (1971) triggered a pulse stretcher with a phototransistor activated by a light-emitting diode; the circuit is able to stretch a 3-microsec input into a 55-msec output pulse. Erickson (1970) and Salomon (1971) both constructed pulse stretchers that utilized one-shot integrated circuits ( $\mu \mathrm{L} 910$ and $\mu \mathrm{L} 9314$, respectively).

The interface module described in this paper combines the convenience of an integrated circuit pulse lengthener-stretcher, featuring a variable-duration output pulse, with an integrated circuit optical isolator interface. The inclusion of the optical isolator extends the possible areas of application. It alone makes it feasible to interface devices that use different voltage levels and polarities.

\section{CONSTRUCTION}

Figure 1 presents a schematic diagram of the circuitry. An incoming signal (positive going) is fed to the

The author appreciates the technical assistance of Laurent Boulet. Requests for reprints should be sent to Louis A. Chiodo, Psychology Laboratory, 400 Old Main, Wayne State University, Detroit, Michigan 48202.

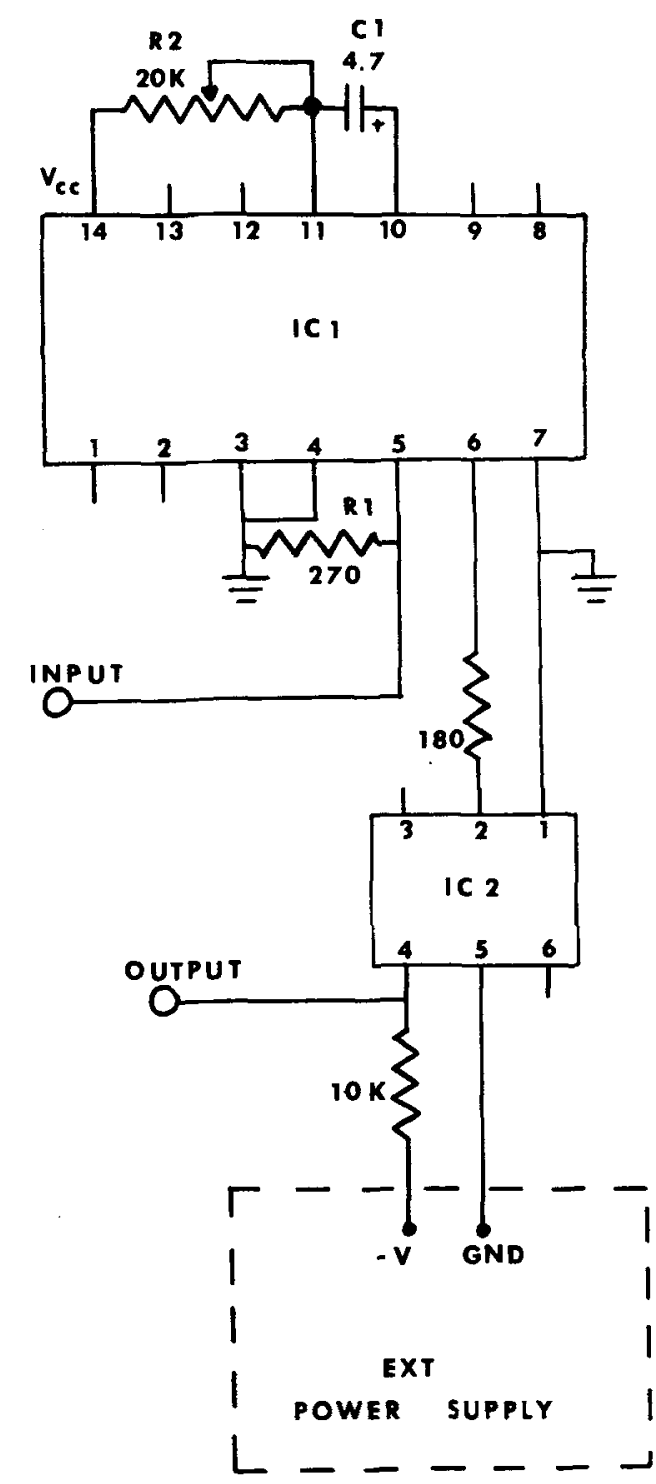

Figure 1. Schematic of the interface module circuit. ICl is a DM 74121 monostable multivibrator and IC2 is a 4 N28 optical coupler/isolator. All resistors are in ohms $(1 / 2 \mathrm{~W}) . \mathrm{Cl}$ is an electrolytic capacitor. 

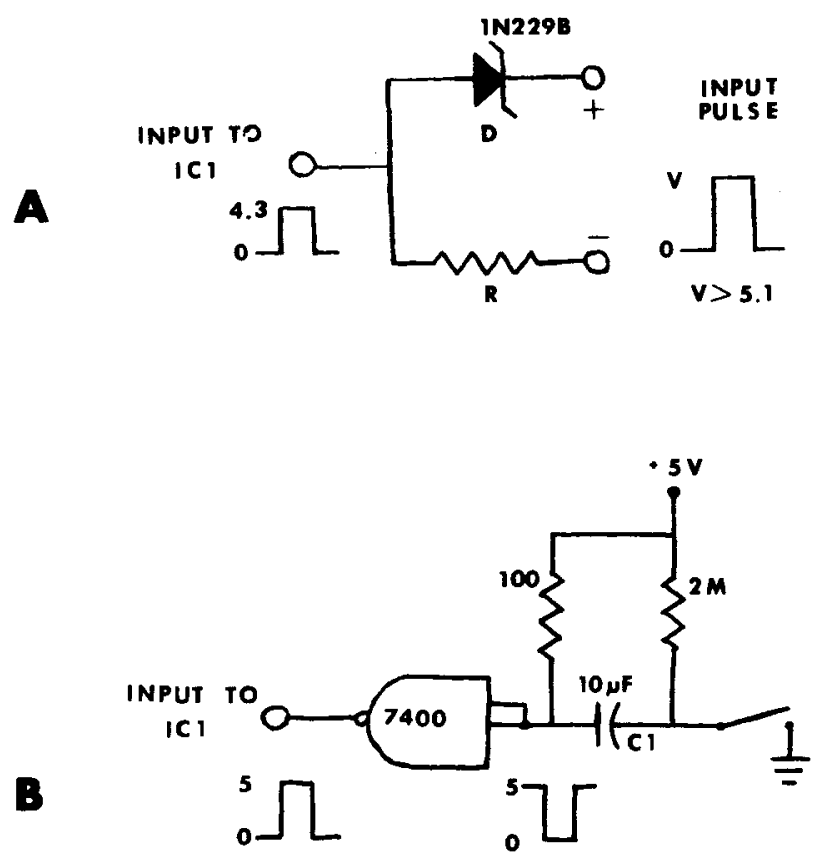

Figure 2. The "clamping" of a limiting diode to the input of ICl (A). A simple contact bounce suppressor (B). All resistors are in ohms $(1 / 2 \mathrm{~W}) . \mathrm{Cl}$ is an electrolytic capacitor.

input of the 74121 monostable multivibrator (National Semiconductor DM74121 or Texas Instruments SN74121) via pin 5, which is referenced to ground with resistor $\mathrm{R} 1$. This allows the output (pin 6) to deliver a positive going one shot that begins virtually instantan. eously with the input (average propagation delay time of IC1 equals 35 nanosec). Once triggered, the outputs of $\mathrm{ICl}$ are isolated from further transitions on the inputs and are affected only by the timing components $\mathrm{C} 1$ and $\mathrm{R} 2$. With the appropriate timing components, the output pulse width may be varied from 30 nanosec to $40 \mathrm{sec}$. The circuit shown in Figure 1 is designed with $\mathrm{C} 1$ as a 4.7-microfarad electrolytic capacitor and R2 a $20 \mathrm{~K}$ potentiometer; these allow pulse widths to extend up to $.97 \mathrm{sec}$. ICl is capable of being effectively triggered by an input as short as 50 nanosec. If the amplitude of the trigger pulse is below $2.5 \mathrm{~V}$, a single transistor amplifier may be added between pin 5 and the input source. If the amplitude of the input pulse is above $5.1 \mathrm{~V}$, a limiting diode (1N5229B or similar) should be "clamped" to the input of IC1, as shown in Figure 2A. Resistor $R$ should be large enough to protect diode $D$ from excessive power which produces a breakdown and possible destruction of IC1.

The power requirement for this and other TTL integrated circuits is $5.5 \mathrm{~V}$. This circuit may be successfully powered with a 6-V general-purpose battery (Burgess F4BP or similar) that has a projected life of greater than 100 continuous $h$ of operation.
A problem often arises when a switch closure is utilized as the input trigger signal for an integrated circuit. Short "false" pulses (such as electrical noise, contact bounce, etc.) will not allow for reliable triggering of an integrated circuit. Various ways of alleviating this problem have been discussed (Baker, 1971; Fontaine, 1971; Laurino, 1970). Figure 2b shows one such contact bounce suppressor that $I$ have employed in order to obtain a single, well-defined input pulse per every switch closure. This input pulse may be applied directly onto the input of ICI.

The input current for IC 1 is $1 \mathrm{~mA}$ maximum when a supply voltage of $5.5 \mathrm{~V}$ is used. This low current draw almost totally eliminates loading of an existing power supply under normal operating conditions. An input pulse with a rise rate as low as $1 \mathrm{~V} /$ microsec will insure the triggering of IC1.

The one-shot output from IC1 is fed into pin 2 of IC2 (Motorola optical coupler/isolator 4N28), which activates an internal infrared light emitter located between pins 1 and 2. The activation of this diode biases a spectrally matched phototransistor whose emitter and collector are on pins 4 and 5 , respectively. Thus, for each input onto IC 2 , an output pulse, synchronized with and identical in duration to the input, will appear at the transistor output (propagation delay time is typically .07 microsec).

The appropriate voltage parameters (polarity and amplitude) of the logic output are obtained by connecting the power supply, to which the interface module's output is to be applied, onto the output transistor of IC2, as shown in Figure 1. This output transistor should be independent of the circuit powering the diode to allow for true isolation between the devices that utilize different power supplies and logic levels. Proper collector-emitter polarity must be observed. Figure 1 shows the circuit receiving output voltage parameters from a negative power supply (such as Coulbourn Instruments - 12 - or $-28-\mathrm{V}$ power supply) so as to deliver a logic " 1 " output pulse.

The output current rating for IC2 is a moderate 1 to $10 \mathrm{~mA}$, with an average response time of 3 microsec. The characteristic operating frequency is $300 \mathrm{kHz}$ and the isolation voltage $\left(\mathrm{V}_{\text {ISO }}\right)$ is $500 \mathrm{~V}$ minimum. The collector output current $\left(\mathrm{I}_{\mathrm{C}}\right)$ is typically $2.0 \mathrm{~mA}$ (Motorola, 1975).

We are presently employing the pulse lengthener described above to interface a 74-45-1 amplitude analyzer (Frederick Haer \& Company, Ann Arbor) with a Coulbourn Instruments solid state module system. The positive going window pulse $(+5 \mathrm{~V}, .5 \mathrm{msec})$ of the amplitude analyzer serves as the input signal to IC1. The Coulbourn Instruments power supply is connected to IC2, as shown in Figure 1, in order to obtain a logic "1" ( $-12 \mathrm{~V}$ to ground) output pulse from the interface module. This output pulse is then applied directly to the appropriate solid state logic unit which completes the 
interfacing of the two divergent instruments. The Coulbourn system has a nominal rise of $\mathbf{4 0 0}$ nanosec, to which the output pulse width of the module can be set by adjusting $R 2$ and monitoring the pulse width with an oscilloscope. The pulse lengthener has provided reliable, trouble-free operation for several months of daily use.

\section{REFERENCES}

BAKER, L. E. Flip-flop pair synchronizes pulses and floats clocks. Electronics, 1971, 44, 56.

BLIss, J. Applications of phototransistors in Electro-Optic systems. Phoenix, Ariz: Motorola Semiconductors, 1971, AN-508.

ERICKSON, K. Shared one shot simplifies pulse width converter. Electronics, 1970, 43, 102
FonTAINE, G. Gate suppresses pulses from switch contact bounces. Electronics, 1971, 44, 76.

LAURINo, A. J. Single IC pulser eliminates contact bounce. Electronics, 1970, 43, 79.

Motorola, INC. Infrared light emitting diode-phototransistor coupler pair. Phoenix, Ariz: Motorola Semiconductors, 1975, DS2623 R3.

RCA Solid State Division. RCA linear integrated circuits. Somerville, N.J: RCA Corporation, 1970.

SAlomon, P. M. FET multiplies time of IC one shot. Electronics, 1971, 44, 90.

WIDLER, R. J. Operational amplifiers. Santa Clara, Calif: National Semiconductor, 1968, AN-4.

(Received for publication October 3, 1976; revision accepted December $15,1976$. 